\title{
IMPROVED POINCARÉ INEQUALITIES AND SOLUTIONS OF THE DIVERGENCE IN WEIGHTED NORMS
}

\author{
Gabriel Acosta, María E. Cejas, Ricardo G. Durán \\ Universidad de Buenos Aires and IMAS-UBA-CONICET \\ Facultad de Ciencias Exactas y Naturales, Departamento de Matemática \\ Pabellón I, Ciudad Universitaria, 1428 CABA, Argentina; gacosta@dm.uba.ar \\ Universidad Nacional de La Plata and CONICET \\ Facultad de Ciencias Exactas, Departamento de Matemática \\ Calle 50 y 115, 1900 La Plata, Buenos Aires, Argentina; mec.eugenia@gmail.com \\ Universidad de Buenos Aires and IMAS-UBA-CONICET \\ Facultad de Ciencias Exactas y Naturales, Departamento de Matemática \\ Pabellón I, Ciudad Universitaria, 1428 CABA, Argentina; rduran@dm.uba.ar
}

Abstract. The improved Poincaré inequality

$$
\left\|\varphi-\varphi_{\Omega}\right\|_{L^{p}(\Omega)} \leq C\|d \nabla \varphi\|_{L^{p}(\Omega)}
$$

where $\Omega \subset \mathbf{R}^{n}$ is a bounded domain and $d(x)$ is the distance from $x$ to the boundary of $\Omega$, has many applications. In particular, it can be used to obtain a decomposition of functions with vanishing integral into a sum of locally supported functions with the same property. Consequently, it can be used to go from local to global results, i.e., to extend to very general bounded domains results which are known for cubes. For example, this methodology can be used to prove the existence of solutions of the divergence in Sobolev spaces. The goal of this paper is to analyze the generalization of these results to the case of weighted norms. When the weight is in $A_{p}$ the arguments used in the un-weighted case can be extended without great difficulty. However, we will show that the improved Poincaré inequality, as well as its above mentioned applications, can be extended to a more general class of weights.

\section{Introduction}

Estimates in weighted norms for classic operators such as the Hardy-Littlewood maximal function, singular integrals of Calderón-Zygmund type and Riesz fractional integrals have been the object of many papers in the last fifty years. Pioneering papers are $[\mathrm{S} 3, \mathrm{SW}]$, for weights of type $|x|^{\alpha}$, and [M, MW] for more general weights. In particular, in [M], Muckenhoupt characterized the weights for which the one dimensional Hardy-Littlewood maximal operator is continuous, for $1<p<\infty$, introducing the now well known class $A_{p}$. In $[\mathrm{CF}]$, Coifman and Fefferman generalized this result to $n$ dimensions. Later, it was proved that the $A_{p}$ condition is necessary and sufficient also for the continuity of the Hilbert and Riesz transforms (see for example [S1]).

In many applications, particularly in the analysis of partial differential equations, estimates in weighted norms arise naturally (see for example the pioneering paper [FKS]). Many of these results are proved for weights in $A_{p}$, the reason being that the

https://doi.org/10.5186/aasfm.2017.4212

2010 Mathematics Subject Classification: Primary 26D10, 46E35.

Key words: Poincaré inequalities, weights, divergence operator.

This research was supported by Universidad de Buenos Aires under grant 20020120100050 and by ANPCyT, Argentina, under grant PICT 2014-1771. 
proofs involve the use of the Hardy-Littlewood maximal operator or the CalderónZygmund singular integral operators theory. In particular, these classic tools have been used in different proofs of the results that we are going to consider in this paper, namely, the so-called improved Poincaré inequality and the existence of solutions of the divergence in Sobolev spaces.

To be more precise let us first introduce some notation. In this paper we consider. By a weight function we mean a nonnegative measurable function $w$ defined in some domain $\Omega \subset \mathbf{R}^{n}$ with $n \geq 2$, and, for $1 \leq p<\infty$, we define

$$
\|f\|_{L_{w}^{p}(\Omega)}=\left(\int_{\Omega}|f(x)|^{p} w(x) d x\right)^{1 / p} .
$$

Given any measurable $S \subset \mathbf{R}^{n}$, a weight $w$ and a function $\varphi$, we write $w(S)=$ $\int_{S} w(x) d x, \varphi_{S, w}=\frac{1}{w(S)} \int_{S} \varphi(x) w(x) d x$ and $\varphi_{\Omega}=\frac{1}{|\Omega|} \int_{\Omega} \varphi(x) d x$, whenever these integrals make sense. If $w$ is such that $L_{w}^{p}(\Omega) \subset L^{1}(\Omega)$, which is the case when $w^{\prime}:=w^{-1 / p-1} \in L^{1}(\Omega)$, we denote with $L_{w, 0}^{p}(\Omega)$ the subspace of functions in $L_{w}^{p}(\Omega)$ with vanishing mean value. In the un-weighted case, for a bounded $\Omega$, we write $L_{0}^{p}(\Omega)$ for the corresponding subspace of $L^{p}(\Omega)$. We say that $w$ is a doubling weight if it is locally integrable and there is a constant $M>0$ such that $w(2 Q) \leq M w(Q)$ for all cubes $Q$, where $2 Q$ is the cube with the same center as $Q$ and twice the side of $Q$.

We will make use of a Whitney decomposition of an open set $\Omega \subseteq \mathbf{R}^{n}$ (see [S2]). That is, there exists a family of cubes $\mathcal{W}=\{Q\}$, with disjoint interiors, and a constant $N>0$ such that,

- $\Omega=\bigcup Q=\bigcup Q^{*}$

- $\operatorname{diam}(Q) \leq \operatorname{dist}(Q, \partial \Omega) \leq 4 \operatorname{diam}(Q)$

- $\sum \chi_{Q^{*}} \leq N \chi_{\Omega}$

where $Q^{*}$ is certain expanded cube of $Q$. We will say that a cube $Q \subset \Omega$ is of Whitney type if

$$
\operatorname{diam}(Q) \approx \operatorname{dist}(Q, \partial \Omega)
$$

Here and in what follows we use the notation $A \approx B$ which means that there exist positive constants $C_{1}$ and $C_{2}$ such that $C_{1} A \leq B \leq C_{2} A$. Let us recall that the $Q^{*}$ can be selected as Whitney type cubes. Finally, by $C$ we will denote a generic constant which can change its value even in the same line.

Given a bounded domain $\Omega$ we denote with $d(x)$ the distance from $x$ to the boundary. Then, the improved Poincaré inequality in the un-weighted case reads as follows. For $1 \leq p<\infty$, there exists a constant $C$ such that,

$$
\left\|\varphi-\varphi_{\Omega}\right\|_{L^{p}(\Omega)} \leq C\|d \nabla \varphi\|_{L^{p}(\Omega)} .
$$

Many proofs of this result have been given under different assumptions on the domain $\Omega$. For example, in [BS], using compactness arguments, the authors proved (1.1) for Lipschitz domains. Later, in [H1], using a more constructive approach based on Whitney decompositions, the result was generalized to John domains. This class of domains was first considered by John, in his work on elasticity [J], and was named after him by Martio and Sarvas in [MS]. A different argument was given, also for John domains, in [DD], where the proof makes use of the Hardy-Littlewood maximal operator.

By classical functional analysis arguments one can show that inequality (1.1) has the following dual version (this is a particular case of results given in [DMRT]). For $1 \leq p<\infty$, if $p^{\prime}$ is the conjugate exponent of $p$ then, there exists a constant $C$ such 
that, given $g \in L_{0}^{p^{\prime}}(\Omega)$, there exists $u$ satisfying

$$
\begin{aligned}
\operatorname{div} u & =g \text { in } \Omega, \\
\left\|\frac{u}{d}\right\|_{L^{p^{\prime}}(\Omega)} & \leq C\|g\|_{L^{p^{\prime}}(\Omega)} .
\end{aligned}
$$

On the other hand, in [DMRT] it was shown that, for $1<p<\infty,(1.2)$ is equivalent to the existence of a decomposition of a function with vanishing integral as a sum of locally supported functions with the same property, namely, numerating the Whitney expanded cubes introduced above, for any $g \in L_{0}^{p^{\prime}}(\Omega)$ there exist $g_{j} \in L_{0}^{p^{\prime}}(\Omega)$ such that $\operatorname{supp} g_{j} \subset Q_{j}^{*}$,

$$
g=\sum_{j} g_{j} \quad \text { and } \quad\|g\|_{L^{p^{\prime}}(\Omega)}^{p^{\prime}} \approx \sum_{j}\left\|g_{j}\right\|_{L^{p^{\prime}}\left(Q_{j}^{*}\right)}^{p}
$$

This decomposition has interesting applications, for example, it can be used to prove the following classic result. Denoting with $D u$ the differential matrix of a vector field $u$, given $g \in L_{0}^{p^{\prime}}(\Omega)$, there exists $u \in W_{0}^{1, p^{\prime}}(\Omega)$ such that,

$$
\begin{aligned}
\operatorname{div} u & =g \text { in } \Omega \\
u & =0 \text { on } \partial \Omega \\
\|D u\|_{L^{p^{\prime}}(\Omega)} & \leq C\|g\|_{L^{p^{\prime}}(\Omega)} .
\end{aligned}
$$

This result has many applications, in particular, it is fundamental in the analysis of the Stokes equations. Let us mention that (1.3) was also proved in [DRS] using a different argument. In that paper the authors extended the decomposition, and consequently (1.4), to weighted norms. Since there arguments are based on the Hardy-Littlewood maximal operator, their results require that the weights be in $A_{p}$.

A natural question that we address in this paper is whether it is possible to generalize the above results for a class of weights more general than $A_{p}$. With this goal we first consider, for $1 \leq p<\infty$, the weighted improved Poincaré inequality which generalizes (1.1), namely,

$$
\left\|\varphi-\varphi_{\Omega, w}\right\|_{L_{w}^{p}(\Omega)} \leq C\|d \nabla \varphi\|_{L_{w}^{p}(\Omega)} .
$$

It is known that this inequality is valid for bounded John domains if $w \in A_{p}$ (see [DD]). As we will see, this result can be extended for more general weights. For example, for a class of weights introduced in [FKS] where the authors consider the classic Poincaré inequality in weighted norms,

$$
\left\|\varphi-\varphi_{\Omega, w}\right\|_{L_{w}^{p}(\Omega)} \leq C\|\nabla \varphi\|_{L_{w}^{p}(\Omega)}
$$

as well as some weighted Sobolev-Poincaré type inequalities.

Apart from proving (1.6) for $A_{p}$ weights, in [FKS] the authors consider, for $p=2$, a class of weights which are not in $A_{2}$. Their technique is based on quasi-conformal mappings, indeed, the authors proved (1.6) when $\Omega$ is a ball, $n \geq 3, p=2$, and $w(x)=J f(x)^{1-\frac{2}{n}}$, where $J f$ is the jacobian of a quasi-conformal mapping $f$. As a particular interesting case of their results they obtained (1.6) for $w(x)=|x|^{\alpha}$ with any $\alpha>0$. Let us recall that this weight is not in $A_{2}$ if $\alpha \geq n$. We will show that (1.5) (and consequently (1.6)) is also valid for the weights considered in [FKS] and, more generally, for $\Omega$ a bounded John domain and $w(x)=J f(x)^{1-\frac{p}{n}}$ when $1 \leq p<n$. In particular, the result is valid for $w(x)=|x|^{\alpha}$ for any $\alpha>0$. 
Moreover, as an application of our results, we prove (1.5) for positive power weights without using quasi-conformal mappings and for $1 \leq p<\infty$, obtaining consequently a different proof of (1.6) and removing the restriction in $p$.

It is now well known that Poincaré type estimates are valid for very general bounded domains whenever they hold in balls or cubes. Indeed, this was proved in [C, H2] with arguments based on [IN]. For example, inequality (1.6) was proved in $[\mathrm{C}]$ for weights in $A_{p}$ where $\Omega$ satisfies the Boman chain condition. As it is known, for bounded domains, this condition is equivalent to being a John domain (see [BKL]). In [H2] (1.6) is extended to a more general class of weights. On the other hand, the proof of the improved Poincaré inequality (1.1) in [H1] mentioned above, uses similar arguments to those in [C, H2].

Roughly speaking, our first theorem says that the weighted improved Poincaré inequality (1.5) is valid in John domains whenever the weighted classic Poincaré inequality holds for Whitney type cubes and $w$ is doubling. The argument of our proof is essentially contained in [C, H2, IN] but, as far as we know, the result has not been written in the way we are doing here, and this is why we include details.

Once we have the generalization of the improved Poincaré inequality we analyze its relation with a decomposition like (1.3) but for the case of weighted norms. It turns out that this relation is valid for a very general class of weights. Finally we apply this decomposition to obtain a weighted version of (1.4) for some weights which are not necessarily in $A_{p}$.

The rest of the paper is organized as follows. In Section 2 we prove the weighted improved Poincaré inequality (1.5) for John domains. Next, in Section 3 we give examples of weights $w \notin A_{p}$ for which the inequality (1.5) holds. In particular, we generalize for $1 \leq p<n$ the arguments given in [FKS]. In Section 4 , we analyze the relation between the improved Poincaré inequality and the decomposition of functions in the weighted case. Finally, in Section 5 we apply that decomposition to prove the existence of solutions of the divergence in weighted Sobolev spaces for power type weights which are not necessarily in $A_{\infty}$.

\section{Weighted improved Poincaré inequality}

The main goal of this section is to prove (1.5) for a wide class of weights. If $\Omega$ is a John domain one can choose a Whitney decomposition satisfying also the following property (see $[\mathrm{H} 1, \mathrm{DRS}]$ ). There exist an open cube $Q_{0}^{*}$ (called central cube) that can be connected with every cube $Q^{*}$ by a finite chain of cubes, $Q_{0}^{*}, Q_{1}^{*}, \ldots, Q_{s}^{*}=Q^{*}$, such that for every $j=0,1, \ldots, s-1$

$$
Q^{*} \subseteq N Q_{j}^{*}
$$

and there exists a cube $R_{j}$ such that

$$
R_{j} \subset Q_{j}^{*} \cap Q_{j+1}^{*} \text { and } Q_{j}^{*} \cup Q_{j+1}^{*} \subset N R_{j} .
$$

Our argument makes use of the following known result.

Lemma 2.1. Let $\mathcal{V}=\{Q\}$ be an arbitrary family of cubes in $\mathbf{R}^{n}$. If $w$ is a doubling weight, $1 \leq p<\infty, N \geq 1$ and $A_{Q}$ are nonnegative real numbers, then

$$
\left\|\sum_{Q \in \mathcal{V}} A_{Q} \chi_{N Q}(x)\right\|_{L_{w}^{p}} \leq C\left\|\sum_{Q \in \mathcal{V}} A_{Q} \chi_{Q}(x)\right\|_{L_{w}^{p}}
$$

where the constant $C$ depends only on $n, N, p$ and $w$. 
Proof. See Lemma 2.3 in [StW, Page 299].

The proof of the next theorem follows the arguments given in [H1, Theorem1.3].

Theorem 2.2. Let $\Omega$ be a bounded John domain and $w \in L^{1}(\Omega)$ be a doubling weight satisfying

$$
\left\|\varphi-\varphi_{Q, w}\right\|_{L_{w}^{p}(Q)} \leq C \operatorname{diam}(Q)\|\nabla \varphi\|_{L_{w}^{p}(Q)}
$$

for all $\varphi \in C^{1}(\bar{Q})$ and all Whitney type cube $Q \subset \Omega$, where $C$ is a constant that does not depend on the cube. Then, for $1 \leq p<\infty$ and all $\varphi \in C^{1}(\Omega)$,

$$
\left\|\varphi-\varphi_{\Omega, w}\right\|_{L_{w}^{p}(\Omega)} \leq C\|d \nabla \varphi\|_{L_{w}^{p}(\Omega)} .
$$

Proof. Let $\mathcal{W}=\{Q\}$ be a Whitney decomposition satisfying the properties described in the previous section. Let us observe that,

$$
\max \left\{w\left(Q_{j}^{*}\right), w\left(Q_{j+1}^{*}\right)\right\} \leq C w\left(Q_{j}^{*} \cap Q_{j+1}^{*}\right)
$$

$j=0,1, \ldots, s-1$ cubes from the chain associated with $Q^{*}$. Since $w \in L^{1}(\Omega)$ it is enough to prove

$$
\left\|\varphi-\varphi_{Q_{0}^{*}, w}\right\|_{L_{w}^{p}(\Omega)} \leq C\|d \nabla \varphi\|_{L_{w}^{p}(\Omega)}
$$

where $Q_{0}^{*}$ is the central cube. We have

$$
\begin{aligned}
& \int_{\Omega}\left|\varphi(x)-\varphi_{Q_{0}^{*}, w}\right|^{p} w(x) d x \\
& \leq 2^{p} \sum_{Q \in \mathcal{W}} \int_{Q^{*}}\left|\varphi(x)-\varphi_{Q^{*}, w}\right|^{p} w(x) d x+2^{p} \sum_{Q \in \mathcal{W}} \int_{Q^{*}}\left|\varphi_{Q_{0}^{*}, w}-\varphi_{Q^{*}, w}\right|^{p} w(x) d x .
\end{aligned}
$$

To estimate the first sum we use (2.2) and that the cubes $Q^{*}$ are of Whitney type,

$$
\begin{aligned}
\sum_{Q \in \mathcal{W}} \int_{Q^{*}}\left|\varphi(x)-\varphi_{Q^{*}, w}\right|^{p} w(x) d x & \leq \sum_{Q \in \mathcal{W}} C \operatorname{diam}\left(Q^{*}\right)^{p} \int_{Q^{*}}|\nabla \varphi(x)|^{p} w(x) d x \\
& \leq C \sum_{Q \in \mathcal{W}} \int_{Q^{*}}|\nabla \varphi(x)|^{p} d(x)^{p} w(x) d x \\
& =C \int_{\Omega}|\nabla \varphi(x)|^{p} d(x)^{p} w(x) d x
\end{aligned}
$$

where for the last inequality we have used that $\sum \chi_{Q^{*}} \leq N \chi_{\Omega}$.

Now, we estimate the second sum. We have

$$
\left|\varphi_{Q_{0}^{*}, w}-\varphi_{Q^{*}, w}\right| \leq \sum_{j=0}^{s-1}\left|\varphi_{Q_{j}^{*}, w}-\varphi_{Q_{j+1}^{*}, w}\right|
$$

using again $\sum \chi_{Q^{*}} \leq N \chi_{\Omega}$, the triangle inequality, (2.2) and that the cubes $Q_{j}^{*}$ are of Whitney type, we obtain

$$
\begin{aligned}
\left|\varphi_{Q_{j}^{*}, w}-\varphi_{Q_{j+1}^{*}, w}\right|^{p} & =\frac{1}{w\left(Q_{j}^{*} \cap Q_{j+1}^{*}\right)} \int_{Q_{j}^{*} \cap Q_{j+1}^{*}}\left|\varphi_{Q_{j}^{*}, w}-\varphi_{Q_{j+1}^{*}, w}\right|^{p} w(y) d y \\
& \leq 2^{p} C \sum_{\alpha=j}^{j+1} \frac{1}{w\left(Q_{\alpha}^{*}\right)} \int_{Q_{\alpha}^{*}}|\nabla \varphi(y)|^{p} d(y)^{p} w(y) d y
\end{aligned}
$$


Since $Q^{*} \subseteq N Q_{\alpha}^{*}$ for $0 \leq \alpha \leq s$, we have

$$
\left|\varphi_{Q_{j}^{*}, w}-\varphi_{Q_{j+1}^{*}, w}\right|^{p} \chi_{Q^{*}}(x) \leq C \sum_{\alpha=j}^{j+1} \frac{\chi_{N Q_{\alpha}^{*}}(x)}{w\left(Q_{\alpha}^{*}\right)} \int_{Q_{\alpha}^{*}}|\nabla \varphi(y)|^{p} d(y)^{p} w(y) d y
$$

and therefore,

$$
\begin{aligned}
& \left|\varphi_{Q_{0}^{*}, w}-\varphi_{Q^{*}, w}\right| \chi_{Q^{*}}(x) \leq C \sum_{j=0}^{s-1}\left(\sum_{\alpha=j}^{j+1} \frac{\chi_{N Q_{\alpha}^{*}}(x)}{w\left(Q_{\alpha}^{*}\right)} \int_{Q_{\alpha}^{*}}|\nabla \varphi(y)|^{p} d(y)^{p} w(y) d y\right)^{1 / p} \\
& \leq C \sum_{R \in \mathcal{W}} \chi_{N R^{*}}(x)\left(\frac{1}{w\left(R^{*}\right)} \int_{R^{*}}|\nabla \varphi(y)|^{p} d(y)^{p} w(y) d y\right)^{1 / p}
\end{aligned}
$$

then,

$$
\begin{aligned}
& \sum_{Q \in \mathcal{W}} \int_{Q^{*}}\left|\varphi_{Q_{0}^{*}, w}-\varphi_{Q^{*}, w}\right|^{p} w(x) d x \leq \sum_{Q \in \mathcal{W}} \int_{Q^{*}}\left|\varphi_{Q_{0}^{*}, w}-\varphi_{Q^{*}, w}\right|^{p} \chi_{Q^{*}}(x) w(x) d x \\
& \leq C \sum_{Q \in \mathcal{W}} \int_{Q^{*}}\left|\sum_{R \in \mathcal{W}}\left(\frac{1}{w\left(R^{*}\right)} \int_{R^{*}}|\nabla \varphi(y)|^{p} d(y)^{p} w(y) d y\right)^{1 / p} \chi_{N R^{*}}(x)\right|^{p} w(x) d x \\
& \leq C \int_{\mathbf{R}^{n}}\left|\sum_{R \in \mathcal{W}}\left(\frac{1}{w\left(R^{*}\right)} \int_{R^{*}}|\nabla \varphi(y)|^{p} d(y)^{p} w(y) d y\right)^{1 / p} \chi_{N R^{*}}(x)\right|^{p} w(x) d x
\end{aligned}
$$

and using now $(2.1)$ and $\sum_{R \in \mathcal{W}} \chi_{R^{*}}(x) \leq N \chi_{\Omega}(x)$ we obtain

$$
\begin{aligned}
& \sum_{Q \in \mathcal{W}} \int_{Q^{*}}\left|\varphi_{Q_{0}^{*}, w}-\varphi_{Q^{*}, w}\right|^{p} w(x) d x \\
& \leq C \int_{\mathbf{R}^{n}}\left|\sum_{R \in \mathcal{W}}\left(\frac{1}{w\left(R^{*}\right)} \int_{R^{*}}|\nabla \varphi(y)|^{p} d(y)^{p} w(y) d y\right)^{1 / p} \chi_{R^{*}}(x)\right|^{p} w(x) d x \\
& \leq C \int_{\mathbf{R}^{n}} \sum_{R \in \mathcal{W}}\left(\frac{1}{w\left(R^{*}\right)} \int_{R^{*}}|\nabla \varphi(y)|^{p} d(y)^{p} w(y) d y\right) \chi_{R^{*}}(x) w(x) d x \\
& =C \sum_{R \in \mathcal{W}} \int_{\mathbf{R}^{n}} \frac{1}{w\left(R^{*}\right)} \chi_{R^{*}}(x) w(x) d x \int_{R^{*}}|\nabla \varphi(y)|^{p} d(y)^{p} w(y) d y \\
& =C \sum_{R \in \mathcal{W}} \int_{R^{*}}|\nabla \varphi(y)|^{p} d(y)^{p} w(y) d y \leq C \int_{\Omega}|\nabla \varphi(y)|^{p} d(y)^{p} w(y) d y
\end{aligned}
$$

concluding the proof.

Remark 2.3. It is known that (2.2) holds for $A_{p}$ weights (this is proved in [FKS]). Moreover, in [C, Theorem 2.14], Chua gives a more general sufficient condition. Indeed, he proves that (2.2) holds for any doubling weight $w$ which satisfies the following condition: there exists $r>1$ such that for all cube $Q_{0} \subset \mathbf{R}^{n}$,

$$
\sup _{Q \subset Q_{0}}|Q|^{1 / n}\left(\frac{1}{|Q|} \int_{Q} w^{r}\right)^{1 / p r}\left(\frac{1}{|Q|} \int_{Q} w^{-r /(p-1)}\right)^{1 / p^{\prime} r}<\infty .
$$

Actually, Chua does not give an explicit expression for the constant in (2.2) but it can be obtained using a standard scaling argument. 


\section{Examples}

The goal of this section is to give some examples which show that the class of weights satisfying the conditions of Theorem 2.2 is larger than $A_{p}$.

Example 3.1. Consider the weights

$$
w(x)=(1+|x|)^{\delta} \prod_{i=1}^{m}\left[\frac{\left|x-a_{i}\right|}{1+\left|x-a_{i}\right|}\right]^{\gamma_{i}} v(x)
$$

where $\delta \geq 0, \gamma_{i} \geq 0,\left\{a_{i}\right\}_{i=1}^{m}$ are points in $\mathbf{R}^{n}, a_{i} \neq a_{j}$ if $i \neq j$, and $v \in A_{p}$. These weights belong to $A_{\infty}$ (see [StW]) but, in general, they do not belong to $A_{p}$. It was proved in $[\mathrm{CW}]$ that they satisfy the inequality $(2.2)$.

Example 3.2. Let $\Gamma$ be a closed subset of $\partial \Omega$ and $d_{\Gamma}(x)$ be the distance from $x$ to $\Gamma$. Define $w(x)=d_{\Gamma}(x)^{\alpha}$, for $\alpha>0$. It is easy to show that (2.2) follows from the classic un-weighted Poincaré inequality. Indeed, if $Q$ is a Whitney type cube we have, for $x, y \in Q$,

$$
d_{\Gamma}(y) \leq|x-y|+d_{\Gamma}(x) \leq \operatorname{diam}(Q)+d_{\Gamma}(x) \approx d(x)+d_{\Gamma}(x) \leq 2 d_{\Gamma}(x),
$$

and therefore, $w$ behaves like a constant in $Q$.

An interesting application is obtained considering a bounded John domain $\Omega$ such that $0 \in \Omega$. Considering $\tilde{\Omega}:=\Omega \backslash\{0\}$ and $w$ as above with $\Gamma=\{0\}$, we have $d_{\Gamma}(x)=|x|$. Then, $w$ is in $L^{1}(\tilde{\Omega})$ and is doubling. Therefore, applying Theorem 2.2 in $\tilde{\Omega}$ we obtain, for $1 \leq p<\infty$,

$$
\int_{\tilde{\Omega}}\left|\varphi(x)-\varphi_{\tilde{\Omega},|x|^{\alpha}}\right|^{p}|x|^{\alpha} d x \leq C \int_{\tilde{\Omega}}|\nabla \varphi(x)|^{p}|x|^{\alpha} \operatorname{dist}(x, \partial \tilde{\Omega})^{p} d x
$$

but we can obviously replace $\tilde{\Omega}$ by $\Omega$ in the integrals, and using that $\operatorname{dist}(x, \partial \tilde{\Omega}) \leq$ $d(x)$, we obtain

$$
\left\|\varphi-\varphi_{\Omega,|x|^{\alpha}}\right\|_{L_{|x|^{\alpha}}^{p}(\Omega)} \leq C\|d \nabla \varphi\|_{L_{|x|^{\alpha}}^{p}(\Omega)} .
$$

Below we will use this estimate for $\alpha>-n$ (the case $-n<\alpha \leq 0$ is known because for those values of $\alpha$ the weight $|x|^{\alpha}$ is in $A_{p}$ ).

Example 3.3. The weighted Poincaré inequality (3.2) in balls was proved for $p=2$ and $n \geq 3$, with a totally different argument, by Fabes, Kenig and Serapioni in [FKS]. Indeed, they showed in that paper that $w(x)=|x|^{\alpha}$, for $\alpha>0$, is a particular case of a general class of weights introduced there, for which the weighted Poincaré inequality in balls holds (although, as this example shows, their weights are not necessarily in $A_{p}$ ). One can trivially change balls by cubes in their arguments and consequently Theorem 2.2 applies for this class of weights.

Here we extend their results for any $n \geq 2$ and $p<n$. This extension is straightforward but we include details for completeness. Actually, our argument is simpler because we are interested only in Poincaré inequality while in [FKS] the authors proved Sobolev-Poincaré inequalities.

Let $f: \mathbf{R}^{n} \rightarrow \mathbf{R}^{n}$ be a quasi-conformal mapping, that is, $f$ is a homeomorphism, the components $f_{i}$ of $f$ have distributional derivatives in $L^{n}(\Omega)$, and there is a constant $M>0$ such that, almost everywhere,

$$
|D f(x)|:=\left(\sum_{i, j}\left(\frac{\partial f_{i}}{\partial x_{j}}(x)\right)^{2}\right)^{1 / 2} \leq M J f(x)^{1 / n}
$$


where $J f$ is the absolute value of the determinant of $D f$.

Lemma 3.4. Given $p$ such that $1 \leq p<n$, define $w(x)=J f(x)^{1-p / n}$. There exists a constant $C$ such that for all cube $Q$ and all $\varphi \in C^{1}(\bar{Q})$

$$
\left\|\varphi-\varphi_{Q, w}\right\|_{L_{w}^{p}(Q)} \leq C \operatorname{diam}(Q)\|\nabla \varphi\|_{L_{w}^{p}(Q)}
$$

Proof. Since $w \in L^{1}(\Omega)$ it is enough to prove the inequality (3.4) replacing $\varphi_{Q, w}$ by a constant $c_{Q}$. The main idea of the proof is to reduce the weighted inequality to an unweighted one by making the change of variables $y=f(x)$.

Observe that

$$
\operatorname{diam}(Q)=C|Q|^{1 / n}=C\left(\int_{Q} d x\right)^{1 / n}=C\left(\int_{f(Q)} J f^{-1}(y) d y\right)^{1 / n} .
$$

Then, using the Hölder inequality with exponents $n /(n-p)$ and its dual $n / p$ we have,

$$
\begin{aligned}
& \int_{Q}\left|\varphi(x)-c_{Q}\right|^{p} w(x) d x=\int_{f(Q)}\left|\left(\varphi \circ f^{-1}\right)(y)-c_{Q}\right|^{p} J f^{-1}(y)^{p / n} d y \\
& \leq\left(\int_{f(Q)}\left|\left(\varphi \circ f^{-1}\right)(y)-c_{Q}\right|^{p *} d y\right)^{p / p *}\left(\int_{f(Q)} J f^{-1}(y) d y\right)^{p / n} \\
& \leq C \operatorname{diam}(Q)^{p}\left(\int_{f(Q)}\left|\left(\varphi \circ f^{-1}\right)(y)-c_{Q}\right|^{p *} d y\right)^{p / p *}
\end{aligned}
$$

where we have used the standard notation for the Sobolev-Poincaré exponent $p *=$ $p n /(n-p)$.

Now, it follows from the condition (3.3) for $f^{-1}$, that

$$
\int_{f(Q)}\left|\nabla\left(\varphi \circ f^{-1}\right)(y)\right|^{p} d y \leq C \int_{Q}|\nabla \varphi(x)|^{p} w(x) d x
$$

and therefore, it is enough to prove

$$
\left(\int_{f(Q)}\left|\left(\varphi \circ f^{-1}\right)(y)-c_{Q}\right|^{p *} d y\right)^{1 / p *} \leq C\left(\int_{f(Q)}\left|\nabla\left(\varphi \circ f^{-1}\right)(y)\right|^{p}\right)^{1 / p}
$$

But, $f(Q)$ is a John domain, and the Sobolev-Poincaré inequality (3.5) for this kind of domains was proved in [B]. Moreover, the constant $C$ in (3.5) depends only on $n$ and the John constant of $f(Q)$ (this is proved, for example, in [DD]) which, according to Lemma 2.3 in [HK, page 539], depends only on $n$ and the $A_{\infty}$ constant of $J f$. Finally, it was proved in $[\mathrm{G}]$ that the $A_{\infty}$ constant of $J f$ depends only on $M$ and $n$ (for the last observation recall that we are assuming that $f$ is quasi-conformal in $\left.\mathbf{R}^{n}\right)$.

Consequently we obtain the following result.

Theorem 3.5. Let $\Omega$ be a bounded John domain and $w(x)=J f(x)^{1-p / n}, 1 \leq$ $p<n$, with $f: \mathbf{R}^{n} \rightarrow \mathbf{R}^{n}$ a quasi-conformal mapping. Then the following inequality holds

$$
\left\|\varphi-\varphi_{\Omega, w}\right\|_{L_{w}^{p}(\Omega)} \leq C\|d \nabla \varphi\|_{L_{w}^{p}(\Omega)} .
$$

Proof. It is known from the results in [G] that $w \in A_{\infty}$, and so, it is doubling. Therefore, in view of (3.4), the result follows from Theorem 2.2.

Remark 3.6. Following [FKS], given $\alpha>0$, we can take $f(x)=|x|^{\beta} x$ with $\beta=\frac{\alpha}{n-p}$ to obtain (3.2) for $1 \leq p<n$ (see [FKS] for details). 


\section{Decomposition of functions of vanishing integral}

The improved Poincaré inequality (1.5) has many applications. In particular, it was shown in [DMRT], that it is related with a useful decomposition for functions of vanishing integral in $\Omega$ as a sum of locally supported functions with the same property. An interesting application of this decomposition is the solvability of the divergence in Sobolev spaces (see [DMRT, DRS]).

The goal of this section is to extend the arguments given in [DMRT] and to show that solutions of the divergence in weighted Sobolev spaces can be obtained for some weights which are not in $A_{p}$. We start with the following lemma which generalizes a result of [DMRT].

Recall that $w^{\prime}:=w^{-1 /(p-1)}$ and that, if $w^{\prime} \in L^{1}(\Omega)$ then $L_{w}^{p} \subset L^{1}(\Omega)$ and therefore the space

$$
L_{w, 0}^{p}(\Omega)=\left\{f \in L_{w}^{p}(\Omega): \int_{\Omega} f=0\right\}
$$

is well defined.

Lemma 4.1. Let $\Omega \subset \mathbf{R}^{n}$ be a bounded domain, and $1<p<\infty,\left\{Q_{j}\right\}$ a Whitney decomposition of $\Omega$ and $\left\{Q_{j}^{*}\right\}$ expanded cubes of $Q_{j}$ as in Section 1 and let $\left\{\phi_{j}\right\}$ be the usual partition of unity associated with the decomposition (see for example [S2]). Given a weight $w$ such that $w^{\prime} \in L^{1}(\Omega)$, we consider the following properties,

(1) $\left\|h-h_{\Omega, w^{\prime}}\right\|_{L_{w^{\prime}}^{p^{\prime}}(\Omega)} \leq C\|d \nabla h\|_{L_{w^{\prime}}^{p^{\prime}}(\Omega)} \forall h \in L_{w^{\prime}}^{p^{\prime}}(\Omega) \cap C^{1}(\Omega)$ such that $\nabla h \in$ $L_{d^{p^{\prime} w^{\prime}}}^{p^{\prime}}(\Omega)^{n}$.

(2) If $g \in L_{w, 0}^{p}(\Omega)$ there exists $u \in L_{d^{-p} w}^{p}(\Omega)^{n}$ such that

$$
\int_{\Omega} u \cdot \nabla h=\int_{\Omega} g h \quad \forall h \in L_{w^{\prime}}^{p^{\prime}}(\Omega) \cap C^{1}(\Omega) \text { such that } \nabla h \in L_{d^{p^{\prime} w^{\prime}}}^{p^{\prime}}(\Omega)^{n}
$$

and

$$
\left\|\frac{u}{d}\right\|_{L_{w}^{p}(\Omega)} \leq C\|g\|_{L_{w}^{p}(\Omega)}
$$

(3) If $g \in L_{w, 0}^{p}(\Omega)$ there exists a decomposition

$$
g=\sum_{j} g_{j}
$$

with $g_{j} \in L_{w, 0}^{p}(\Omega), \operatorname{supp} g_{j} \subset Q_{j}^{*}$, and

$$
\|g\|_{L_{w}^{p}(\Omega)}^{p} \approx \sum_{j}\left\|g_{j}\right\|_{L_{w}^{p}\left(Q_{j}^{*}\right)}^{p}
$$

Then,

$$
(1) \Leftrightarrow(2) \Rightarrow(3)
$$

Proof. $(1) \Rightarrow(2)$ : Let $S \subset L_{d^{p^{\prime}} w^{\prime}}^{p^{\prime}}(\Omega)^{n}$ be the subspace given by

$$
S=\left\{v \in L_{d^{p^{\prime}} w^{\prime}}^{p^{\prime}}(\Omega)^{n}: v=\nabla h \text { with } h \in L_{w^{\prime}}^{p^{\prime}}(\Omega) \cap C^{1}(\Omega)\right\},
$$


and set $\mathcal{L}(\nabla h)=\int_{\Omega} g h$. Since $\int_{\Omega} g=0, \mathcal{L}$ defines a linear form on $S$. Moreover, it follows from (1) that

$$
\begin{aligned}
|\mathcal{L}(\nabla h)| & =\left|\int_{\Omega} g\left(h-h_{\Omega, w^{\prime}}\right)\right| \leq C\|g\|_{L_{w}^{p}(\Omega)}\left\|h-h_{\Omega, w^{\prime}}\right\|_{L_{w^{\prime}}^{p^{\prime}}(\Omega)} \\
& \leq C\|g\|_{L_{w}^{p}(\Omega)}\|d \nabla h\|_{L_{w^{\prime}}^{p^{\prime}}(\Omega)} .
\end{aligned}
$$

By the Hahn-Banach theorem $\mathcal{L}$ can be extended as a linear continuous functional defined on $L_{d^{p^{\prime} w^{\prime}}}^{p^{\prime}}(\Omega)^{n}$, and therefore, by duality there exists $u \in L_{d^{-p} w}^{p}(\Omega)^{n}$ such that

$$
\mathcal{L}(v)=\int_{\Omega} u \cdot v \quad \text { and } \quad\left\|\frac{u}{d}\right\|_{L_{w}^{p}(\Omega)} \leq C\|g\|_{L_{w}^{p}(\Omega)}
$$

in particular, taking $v=\nabla h \in S$, we obtain (2).

$(2) \Rightarrow(1)$ : Let $h \in L_{w^{\prime}}^{p^{\prime}}(\Omega) \cap C^{1}(\Omega)$ be such that $\nabla h \in L_{d^{p^{\prime} w^{\prime}}}^{p^{\prime}}(\Omega)^{n}$. Then,

$$
\left\|h-h_{\Omega, w^{\prime}}\right\|_{L_{w^{\prime}}^{p^{\prime}}(\Omega)}=\sup _{\|g\|_{L_{w^{\prime}}^{p}(\Omega)=1}} \int_{\Omega}\left(h-h_{\Omega, w^{\prime}}\right) g w^{\prime}=\sup _{\|g\|_{L_{w^{\prime}}^{p}(\Omega)=1}} \int_{\Omega} h\left(g-g_{\Omega, w^{\prime}}\right) w^{\prime}
$$

but, since $\left(g-g_{\Omega, w^{\prime}}\right) w^{\prime} \in L_{w, 0}^{p}(\Omega)$ with

$$
\left\|\left(g-g_{\Omega, w^{\prime}}\right) w^{\prime}\right\|_{L_{w}^{p}(\Omega)}=\left\|g-g_{\Omega, w^{\prime}}\right\|_{L_{w^{\prime}}^{p}(\Omega)} \leq C\|g\|_{L_{w^{\prime}}^{p}(\Omega)}
$$

we know from (2) that there exists $u \in L_{d^{-p_{w}}}^{p}(\Omega)^{n}$ such that

$$
\int_{\Omega} u \cdot \nabla h=\int_{\Omega} h\left(g-g_{\Omega, w^{\prime}}\right) w^{\prime}
$$

satisfying

Therefore,

$$
\left\|\frac{u}{d}\right\|_{L_{w}^{p}(\Omega)} \leq C\left\|\left(g-g_{\Omega, w^{\prime}}\right) w^{\prime}\right\|_{L_{w}^{p}(\Omega)} \leq C\|g\|_{L_{w^{\prime}}^{p}(\Omega)}
$$

$$
\int_{\Omega} h\left(g-g_{\Omega, w^{\prime}}\right) w^{\prime}=\int_{\Omega} u \cdot \nabla h \leq\|d \nabla h\|_{L_{w^{\prime}}^{p^{\prime}}(\Omega)}\left\|\frac{u}{d}\right\|_{L_{w}^{p}(\Omega)} \leq C\|d \nabla h\|_{L_{w^{\prime}}^{p^{\prime}}(\Omega)}\|g\|_{L_{w^{\prime}}^{p}(\Omega)}
$$

and replacing in (4.3) we obtain (1).

$(2) \Rightarrow(3)$ Given $g \in L_{w, 0}^{p}(\Omega)$ let $u \in L_{d^{-p} w}^{p}(\Omega)^{n}$ as in (2). Observe that, in particular, we can take $h \in C_{0}^{\infty}(\Omega)$ in (4.1), and therefore, div $u=g$ in $\Omega$. Then, we define

and so, we have

$$
g_{j}=\operatorname{div}\left(\phi_{j} u\right)
$$

$$
g=\operatorname{div} u=\operatorname{div}\left(u \sum_{j} \phi_{j}\right)=\sum_{j} \operatorname{div}\left(\phi_{j} u\right)=\sum_{j} g_{j} .
$$

Since supp $\phi_{j} \subseteq Q_{j}^{*}$ we have supp $g_{j} \subseteq Q_{j}^{*}$ and $\int g_{j}=0$. Moreover, each point in $\Omega$ is contained in at most $N$ of the cubes $Q_{j}^{*}$, consequently

$$
|g(x)|^{p} \leq C \sum_{j}\left|g_{j}(x)\right|^{p}
$$

and then

$$
\|g\|_{L_{w}^{p}(\Omega)}^{p} \leq C \sum_{j}\left\|g_{j}\right\|_{L_{w}^{p}\left(Q_{j}^{*}\right)}^{p}
$$

where the constant $C$ depends only on $p$ and $n$. 
To prove the other inequality we use that $\left\|\phi_{j}\right\|_{L^{\infty}} \leq 1$ and $\left\|\nabla \phi_{j}\right\|_{L^{\infty}} \leq C / d_{j}$, where $d_{j}$ is the distance of $Q_{j}$ to $\partial \Omega$. Then, we have

$$
\begin{aligned}
\left\|g_{j}\right\|_{L_{w}^{p}\left(Q_{j}^{*}\right)}^{p} & =\int_{Q_{j}^{*}}\left|g_{j}\right|^{p} w=\int_{Q_{j}^{*}}\left|\operatorname{div}\left(\phi_{j} u\right)\right|^{p} w=\int_{Q_{j}^{*}}\left|\nabla \phi_{j} \cdot u+\phi_{j} \operatorname{div} u\right|^{p} w \\
& \leq C\left(\int_{Q_{j}^{*}}\left|\nabla \phi_{j}\right|^{p}|u|^{p} w+\int_{Q_{j}^{*}}\left|\phi_{j}(x)\right|^{p}|\operatorname{div} u|^{p} w\right) \\
& \leq C\left(\left\|\frac{u}{d}\right\|_{L_{w}^{p}\left(Q_{j}^{*}\right)}^{p}+\|g\|_{L_{w}^{p}\left(Q_{j}^{*}\right)}^{p}\right)
\end{aligned}
$$

and therefore, using that $\sum_{j} \chi_{Q_{j}^{*}} \leq N \chi_{\Omega}$, it follows from (4.2) that

$$
\sum_{j}\left\|g_{j}\right\|_{L_{w}^{p}\left(Q_{j}^{*}\right)}^{p} \leq C\|g\|_{L_{w}^{p}(\Omega)}^{p} .
$$

\section{Solvability of the divergence in weighted Sobolev spaces}

As a consequence of the results given in the previous section we obtain a result which generalizes the arguments given in [DMRT, DRS] to show the existence of solutions of the divergence in weighted Sobolev spaces for weights which are not necessarily in $A_{p}$. Roughly speaking, the divergence can be solved in weighted Sobolev spaces in John domains whenever it can be solved in cubes.

Given a domain $\Omega$ and weights $w_{0}$ and $w_{1}$, such that $L_{w_{0}}^{p}(\Omega) \subset L_{l o c}^{1}(\Omega)$, we define

$$
W_{w_{0}, w_{1}}^{1, p}(\Omega)=\left\{v: \Omega \rightarrow \mathbf{R}^{n}: v \in L_{w_{0}}^{p}(\Omega),|D v| \in L_{w_{1}}^{p}(\Omega)\right\},
$$

with its natural norm, and

$$
W_{0, w_{0}, w_{1}}^{1, p}(\Omega)=\overline{C_{0}^{\infty}(\Omega) \cap W_{w_{0}, w_{1}}^{1, p}(\Omega)} .
$$

Theorem 5.1. Given $\Omega \subset \mathbf{R}^{n}$ and $1<p<\infty$. Let $w_{0}$ and $w_{1}$ be weights with $w_{0}^{\prime}, w_{1}^{\prime} \in L^{1}(\Omega)$ and such that the inequality

$$
\left\|h-h_{\Omega, w_{1}^{\prime}}\right\|_{L_{w_{1}^{\prime}}^{p^{\prime}(\Omega)}} \leq C\|d \nabla h\|_{L_{w_{1}^{\prime}}^{p^{\prime}}(\Omega)}
$$

holds for all $h \in L_{w_{1}^{\prime}}^{p^{\prime}}(\Omega) \cap C^{1}(\Omega)$. Assume that, for any $g \in L_{w_{1}, 0}^{p}(Q)$ and any Whitney type cube $Q \subset \Omega$, there exists $u \in W_{0, w_{0}, w_{1}}^{1, p}(Q)$ satisfying

$$
\operatorname{div} u=g \quad \text { in } Q
$$

and

$$
\|u\|_{L_{w_{0}}^{p}(Q)}+\|D u\|_{L_{w_{1}}^{p}(Q)} \leq C\|g\|_{L_{w_{1}}^{p}(Q)}
$$

where the constant $C$ is independent of the cube $Q$. Then, for any $g \in L_{w_{1}, 0}^{p}(\Omega)$ there exists $u \in W_{0, w_{0}, w_{1}}^{1, p}(\Omega)$ such that

$$
\operatorname{div} u=g \quad \text { in } \Omega
$$

and

$$
\|u\|_{L_{w_{0}}^{p}(\Omega)}+\|D u\|_{L_{w_{1}}^{p}(\Omega)} \leq C\|g\|_{L_{w_{1}}^{p}(\Omega)} .
$$

Proof. First of all observe that $w_{0}^{\prime} \in L^{1}(\Omega)$ implies that $L_{w_{0}}^{p}(\Omega) \subset L^{1}(\Omega)$, and therefore, derivatives of functions in $L_{w_{0}}^{p}(\Omega)$ exist in the distributional sense and so the space $W_{w_{0}, w_{1}}^{1, p}(\Omega)$ is well defined. Now, since $w_{1}^{\prime} \in L^{1}(\Omega)$, we can apply Lemma 4.1, 
and so, given $g \in L_{w_{1}, 0}^{p}(\Omega)$ we decompose $g=\sum g_{j}$ as in (3) of that lemma. By our hypothesis, for each $j$, there exists $u_{j} \in W_{0, w_{0}, w_{1}}^{1, p}\left(Q_{j}^{*}\right)$ satisfying

$$
\operatorname{div} u_{j}=g_{j} \quad \text { in } Q_{j}^{*}
$$

and

$$
\left\|u_{j}\right\|_{L_{w_{0}}^{p}\left(Q_{j}^{*}\right)}+\left\|D u_{j}\right\|_{L_{w_{1}}^{p}\left(Q_{j}^{*}\right)} \leq C\left\|g_{j}\right\|_{L_{w_{1}}^{p}\left(Q_{j}^{*}\right)} .
$$

Then, defining $u=\sum_{j} u_{j}$, we have div $u=g$. Moreover,

$$
\begin{aligned}
\|u\|_{L_{w_{0}}^{p}(\Omega)}^{p}+\|D u\|_{L_{w_{1}}^{p}(\Omega)}^{p} & =\int_{\Omega}\left|\sum_{j} u_{j}\right|^{p} w_{0}(x) d x+\int_{\Omega}\left|\sum_{j} D u_{j}\right|^{p} w_{1}(x) d x \\
& \leq C \sum_{j} \int_{Q_{j}^{*}}\left\{\left|u_{j}(x)\right|^{p} w_{0}(x)+\left|D u_{j}(x)\right|^{p} w_{1}(x)\right\} d x \\
& \leq C \sum_{j} \int_{Q_{j}^{*}}\left|g_{j}(x)\right|^{p} w_{1}(x) d x \leq C\|g\|_{L_{w_{1}}^{p}(\Omega)}^{p} .
\end{aligned}
$$

As an example we show that Theorem 5.1 can be applied to solve the divergence equation in power weighted spaces.

Theorem 5.2. Let $\Omega \subset \mathbf{R}^{n}$ be a bounded John domain such that $0 \in \Omega, 1<$ $p<\infty$, and $-\infty<\gamma<n(p-1)$. Given $g \in L_{|x|^{\gamma}, 0}^{p}(\Omega)$ there exists $u \in W_{0,|x|^{\gamma},|x|^{\gamma}}^{1, p}(\Omega)$ such that

$$
\operatorname{div} u=g \quad \text { in } \Omega
$$

and

$$
\|u\|_{L_{|x| \gamma}^{p}(\Omega)}+\|D u\|_{L_{|x| \gamma}^{p}(\Omega)} \leq C\|g\|_{L_{|x| \gamma}^{p}(\Omega)} .
$$

Proof. First of all observe that, if $w(x)=|x|^{\gamma}$, then $w^{\prime}=|x|^{-\frac{\gamma}{p-1}} \in L^{1}(\Omega)$, indeed, from our hypotheses, $-\frac{\gamma}{p-1}>-n$. In particular $L_{|x| \gamma}^{p}(\Omega) \subset L^{1}(\Omega)$ and so $L_{|x|^{\gamma}, 0}^{p}(\Omega)$ and $W_{0,|x|^{\gamma},|x|^{\gamma}}^{1, p}(\Omega)$ are well defined.

Now, if $-n<\gamma<n(p-1)$, the weight $|x|^{\gamma}$ is in $A_{p}$, and therefore, the result was proved in [DRS, page 103]. Although the authors of that paper only state the bound for the second term on the left hand side of (5.1), the estimate for the other term follows immediately by the weighted Poincare inequality (which is known to be valid for $A_{p}$ weights).

On the other hand, for the case $\gamma \leq-n$, we proceed as in Example 3.2 and introduce $\tilde{\Omega}=\Omega \backslash\{0\}$. It is easy to see that, if $\phi \in C_{0}^{\infty}(\Omega) \cap L_{|x| \gamma}^{p}(\Omega)$, then $\phi(0)=0$, and therefore,

$$
W_{0,|x|^{\gamma},|x|^{\gamma}}^{1, p}(\tilde{\Omega})=W_{0,|x|^{\gamma},|x|^{\gamma}}^{1, p}(\Omega) .
$$

Then, we conclude the proof applying Theorem 5.1 in $\tilde{\Omega}$. Indeed, the hypotheses of that theorem, concerning the existence of solutions in Whitney type cubes, are easily verified because the weight restricted to those cubes behaves like a constant (see details in Example 3.2).

In what follows we are going to show that, for $1<p<n$, Theorem 5.2 can be improved replacing the term $\|u\|_{L_{|x| \gamma}^{p}(\Omega)}$ in (5.1) by the stronger one $\|u\|_{L_{\left.|x|^{\gamma}\right|^{p}}^{p}(\Omega)}$. 
To do this we use the technique based in quasi-conformal mappings. Let us remark that this improvement does not follow from the bound obtained for the derivatives of $u$ because there is no constant $C$ such that the inequality

$$
\|\phi\|_{L_{|x| \gamma-p}^{p}(\Omega)} \leq C\|\nabla \phi\|_{L_{|x| \gamma}^{p}(\Omega)},
$$

holds for a general $\phi \in C_{0}^{\infty}(\Omega)$. Indeed, if $-n<\gamma \leq-n+p$ and $\phi$ is equal to one in a neighborhood of 0 , the right hand side is finite while the left one is not.

For $-\infty<\gamma \leq-n$ the result in the following theorem can be proved by the same argument used in Theorem 5.1, and therefore, the interesting case in the following proof is when $-n<\gamma<n(p-1)$. However, since the argument is independent of the value of $\gamma$, we write the proof in the general case.

Theorem 5.3. Let $\Omega \subset \mathbf{R}^{n}$ be a bounded John domain such that $0 \in \Omega, 1<$ $p<n$, and $-\infty<\gamma<n(p-1)$. Given $g \in L_{|x|^{\gamma}, 0}^{p}(\Omega)$ there exists $u \in W_{0,|x|^{\gamma-p},|x|^{\gamma}}^{1, p}$ such that

$$
\operatorname{div} u=g \quad \text { in } \Omega
$$

and

$$
\|u\|_{L_{|x| \gamma-p}^{p}(\Omega)}+\|D u\|_{L_{|x| \gamma}^{p}(\Omega)} \leq C\|g\|_{L_{|x| \gamma}^{p}(\Omega)} .
$$

Proof. We start as in the proof of Theorem 5.2 observing that $L_{|x|^{\gamma}, 0}^{p}(\Omega)$ and $W_{0,|x|^{\gamma-p},|x|^{\gamma}}^{1, p}(\Omega)$ are well defined. Defining $w_{1}(x)=|x|^{\gamma}$ we have $w_{1}^{\prime}(x)=|x|^{\alpha}$ with $\alpha=-\frac{\gamma}{p-1}>-n$ and so, in view of Example 3.2, the weighted improved Poincaré inequality

$$
\left\|h-h_{\Omega, w_{1}^{\prime}}\right\|_{L_{w_{1}^{\prime}}^{p^{\prime}(\Omega)}} \leq C\|d \nabla h\|_{L_{w_{1}^{\prime}}^{p^{\prime}}(\Omega)}
$$

holds. Therefore, the result will be a consequence of Theorem 5.1, if we show the solvability of the divergence in cubes. This will be done in the following lemma.

Lemma 5.4. Let $Q$ be a cube, $1<p<n$, and $-\infty<\gamma<n(p-1)$. Given $g \in L_{|x|^{\gamma}, 0}^{p}(Q)$ there exists $u \in W_{0,|x|^{\gamma-p},|x|^{\gamma}}^{1, p}(Q)$ such that

$$
\operatorname{div} u=g \quad \text { in } Q
$$

and

$$
\|u\|_{L_{|x|^{\gamma-p}}^{p}(Q)}+\|D u\|_{L_{|x| \gamma}^{p}(Q)} \leq C\|g\|_{L_{|x| \gamma}^{p}(Q)} .
$$

Proof. Given $\gamma$ we take $\beta$ such that $\gamma=\beta n(1-p)<n(p-1)$. Since $\gamma<n(p-1)$ we have $\beta>-1$, and therefore, $f(x)=|x|^{\beta} x$ is a quasi-conformal mapping. Using the change of variables $y=f(x)$ we define $h(y)=g(x) J f^{-1}(f(x))$. Then, $h \in L_{0}^{p}(f(Q))$, and therefore, since $f(Q)$ is a John domain (actually, for the particular $f$ considered here it is a Lipschitz domain), we know that there exists $v \in W_{0}^{1, p}(f(Q))$ such that

$$
\operatorname{div} v=h \quad \text { in } f(Q)
$$

and

$$
\int_{f(Q)}|y|^{-p}|v(y)|^{p} d y+\int_{f(Q)}|D v(y)|^{p} d y \leq C \int_{f(Q)}|h(y)|^{p} d y
$$

see, for example, $[\mathrm{ADM}]$ or $[\mathrm{Ga}]$ for the existence of $v$ satisfying $(5.2)$ and $\|D v\|_{L^{p}(f(Q))}$ $\leq C\|h\|_{L^{p}(f(Q))}$. The estimate for the first term on the right hand side of (5.3) is a consequence of

$$
\int_{f(Q)}|y|^{-p}|v(y)|^{p} d y \leq C \int_{f(Q)}|D v(y)|^{p} d y
$$


which can be obtained from the results for fractional integrals given in [SW] (and it is also a particular case of the so-called Caffarelli-Kohn-Nirenberg inequalities [CKN]).

Now, using the so-called Piola transform, we define

$$
u(x)=J f(x) D f^{-1}(f(x)) v(f(x)) .
$$

Then, we have

$$
\operatorname{div} u=g
$$

and, since $J f(x) \approx|x|^{n \beta(1-p)}=|x|^{\gamma}$, we have to prove that

$$
\int_{Q}|D u(x)|^{p} J f(x)^{1-p} d x \leq C \int_{Q}|g(x)|^{p} J f(x)^{1-p} d x
$$

and

$$
\int_{Q}|u(x)|^{p}|x|^{-p} J f(x)^{1-p} \leq C \int_{Q}|g(x)|^{p} J f(x)^{1-p} d x .
$$

Changing variables we have

$$
\int_{f(Q)}|h(y)|^{p} d y=\int_{Q}|g(x)|^{p} J f(x)^{1-p} d x
$$

and therefore, it is enough to show that

$$
\int_{Q}|D u(x)|^{p} J f(x)^{1-p} d x \leq C \int_{f(Q)}|h(y)|^{p} d y
$$

and

$$
\int_{Q}|u(x)|^{p}|x|^{-p} J f(x)^{1-p} d x \leq C \int_{f(Q)}|h(y)|^{p} d y
$$

But, we have

$$
D u(x)=D\left[J f(x) D f^{-1}(f(x))\right] v(f(x))+J f(x) D f^{-1}(f(x)) D v(f(x)) D f(x)
$$

and therefore,

$$
\int_{Q}|D u(x)|^{p} J f(x)^{1-p} d x \leq I+I I
$$

with

$$
I:=\int_{Q}\left|D\left[J f(x) D f^{-1}(f(x))\right]\right|^{p}|v(f(x))|^{p} J f(x)^{1-p} d x
$$

and

$$
I I:=\int_{Q}\left|D f^{-1}(f(x))\right|^{p}|D f(x)|^{p}|D v(f(x))|^{p} J f(x) d x .
$$

But, using that $f$ and $f^{-1}$ are quasi-conformal mappings, and (5.3), we obtain

$$
I I \leq C \int_{Q}|D v(f(x))|^{p} J f(x) d x=C \int_{f(Q)}|D v(y)|^{p} d y \leq C \int_{f(Q)}|h(y)|^{p} d y .
$$

On the other hand we have,

$$
\begin{aligned}
I \leq C\{ & \int_{Q}|D J f(x)|^{p}\left|D f^{-1}(f(x))\right|^{p}|v(f(x))|^{p} J f(x)^{1-p} d x \\
& \left.+\int_{Q}\left|D^{2} f^{-1}(f(x))\right|^{p}|D f(x)|^{p}|v(f(x))|^{p} J f(x) d x\right\} .
\end{aligned}
$$

Now, recalling that $y=f(x)$, it is easy to check that

$$
|D J f(x)| \leq C|x|^{\beta n-1}=|y|^{\frac{\beta n-1}{\beta+1}},
$$


and, using also that $f^{-1}$ is quasi-conformal and that

$$
J f^{-1}(y) \leq C|y|^{-\frac{\beta n}{\beta+1}} \quad \text { and } \quad J f(x)=J f\left(f^{-1}(y)\right) \leq C|y|^{\frac{\beta n}{\beta+1}}
$$

we obtain that the first term on the right hand side of (5.7) is less than or equal to

$$
C \int_{f(Q)}|y|^{\frac{p(\beta n-1)}{\beta+1}}|y|^{-\frac{p \beta}{\beta+1}}|y|^{\frac{\beta n(1-p)}{\beta+1}}|y|^{-\frac{\beta n}{\beta+1}}|v(y)|^{p} d y=C \int_{f(Q)}|y|^{-p}|v(y)|^{p} d y,
$$

Now, for the second term on the right hand side of (5.7), changing variables and using that

$$
\left|D^{2} f^{-1}(y)\right| \leq C|y|^{-\frac{2 \beta+1}{\beta+1}} \quad \text { and } \quad\left|D f\left(f^{-1}(y)\right)\right| \leq C|y|^{\frac{\beta}{\beta+1}}
$$

we obtain

$$
\begin{aligned}
& \int_{Q}\left|D^{2} f^{-1}(f(x))\right|^{p}|D f(x)|^{p}|v(f(x))|^{p} J f(x) d x \\
& =\int_{f(Q)}\left|D^{2} f^{-1}(y)\right|^{p}\left|D f\left(f^{-1}(y)\right)\right|^{p}|v(y)|^{p} d y \leq C \int_{f(Q)}|y|^{-p}|v(y)|^{p} d y .
\end{aligned}
$$

Collecting these estimates and using (5.3) we obtain

$$
I \leq C \int_{f(Q)}|y|^{-p}|v(y)|^{p} d y \leq C \int_{f(Q)}|h(y)|^{p} d y,
$$

and therefore (5.5) is proved.

Finally, from the definition of $u$ given in (5.4) and changing variables, we have

$$
\left.\int_{Q}|u(x)|^{p}|x|^{-p} J f(x)^{1-p} d x=\int_{f(Q)} \mid D f^{-1}(y)\right)\left.\right|^{p}|y|^{-\frac{p}{\beta+1}}|v(y)|^{p} d y
$$

and using that $f^{-1}$ is quasi-conformal, the first estimate in (5.8), and (5.3), we obtain (5.6).

\section{References}

[ADM] Acosta, G., R. DurÁn, and M. A. Muschietti: Solutions of the divergence operator on John domains. - Adv. Math. 206 (2006), no. 2, 373-401.

[BS] Boas, H. B., and E. J. Straube: Integral inequalities of Hardy and Poincaré type. Proc. Amer. Math. Soc. 103, 1988, 172-176.

[B] Bojarski, B.: Remarks on Sobolev imbedding inequalities. - In: Lecture Notes in Math. 1351, Springer-Verlag, Berlin, 1989, 52-68.

[BKL] Buckley, S., P. Koskela, and G. Lu: Boman equals John. - In: XVIth Rolf Nevanlinna Colloquium (Joensuu, 1995), de Gruyter, Berlin (1996), 91-99.

[CKN] Caffarelli, L., R. Kohn, and L. Nirenberg: First order interpolation inequalities with weights. - Compositio Math. 53, 1984, 259-275.

[CW] Chanillo, S., and R. Wheeden: Poincaré inequalities for a class of non- $A_{p}$ weights. Indiana Univ. Math. J. 41, 1992, 605-623.

[C] ChuA, S.-K.: Weighted Sobolev inequalities on domains satisfying the chain condition. Proc. Amer. Math. Soc. 117, 1993, 449-457.

[CF] Colfman, R., and C. Fefferman: Weighted norm inequalities for maximal functions and singular integrals. - Studia Math. 51:3, 1974, 241-250.

[DRS] Diening, L., M. RuŽickA, and K. Schumacher: A decomposition technique for John domains. - Ann. Acad. Sci. Fenn. Math. 35, 2010, 87-114. 
[DD] Drelichman, I., and R. DurÁn: Improved Poincaré inequality with weights. - J. Math. Anal. Appl. 347, 2008, 286-293.

[DMRT] Durán, R., M. A. Muschietti, E. Russ, and P. Tchamitchian: Divergence operator and Poincaré inequalities on arbitrary bounded domains. - Complex Var. Elliptic Equ. $55: 8,2010,795-816$.

[FKS] Fabes, E. B., C. E. Kenig, R. P. SERApioni: The local regularity of solutions of degenerate elliptic equations. - Comm. Partial Differential Equations 7:1, 1982, 77-116.

[Ga] Galdi, G.: An introduction to the mathematical theory of the Navier-Stokes equations. - Springer, New York, 1994.

[G] Gehring, F. W.: The $L^{p}$-integrability of the partial derivatives of a quasi-conformal mapping. - Acta Math. 130, 1973, 266-277.

[HK] Heinonen, J., and P. Koskela: $A_{\infty}$-condition for the jacobian of a quasi-conformal mapping. - Proc. Amer. Math. Soc. 120, 1994, 535-543.

[H1] Hurri-Syrjänen, R.: An improved Poincaré inequality. - Proc. Amer. Math. Soc. 120, 1994, 213-222.

[H2] Hurri-Syrjanen, R.: A weighted Poincaré inequality with a doubling weight. - Proc. Amer. Math. Soc. 126, 1998, 545-552.

[IN] Iwaniec, T., and C. A. Nolder: Hardy-Littlewood inequality for quasiregular mappings in certain domains in $\mathbf{R}^{n}$. - Ann. Acad. Sci. Fenn. Ser. A I Math. 10, 1985, 267-282.

[J] John, F.: Rotation and strain. - Comm. Pure Appl. Math. 14, 1961, 391-413.

[MS] Martio, O., and J. Sarvas: Injectivity theorems in plane and space. - Ann. Acad. Sci. Fenn. Ser. A I Math. 4, 1978/1979, 383-401.

[M] Muckenhoupt, B.: Weighted norme inequalities for the Hardy maximal function. Trans. Amer. Math. Soc. 165, 1972, 207-226.

[MW] Muckenhoupt, B., and R. Wheeden: Weighted norm inequalities for fractional integrals. - Trans. Amer. Math. Soc. 192, 1974, 261-274.

[S1] Stein, E. M.: Harmonic analysis: Real variable-methods, ortogonality, and oscillatory integrals. - Princeton Univ. Press, 1993.

[S2] Stein, E. M.,: Singular integrals and differentiability properties of functions. - Princeton Univ. Press, 1970.

[S3] Stein, E. M.: Note on singular integrals. - Proc. Amer. Math. Soc. 8, 1957, 250-254.

[SW] Stein, E. M., and G. Weiss: Fractional integrals on $n$-dimensional Euclidean space. - J. Math. Mech. 7, 1958, 503-514.

[StW] Stromberg, J., and R. Wheeden: Fractional integrals on weighted $H^{p}$ and $L^{p}$ spaces. - Trans. Amer. Math. Soc. 287:1, 1985, 293-321. 